\title{
L'EFFET DE LA DATE D'ÉCLOSION SUR LE RÉSULTAT DE LA SÉLECTION POUR LA PRÉCOCITÉ SEXUELLE DES VOLAILLES.
}

PAR

\section{P. MERAT}

Station de Recherches Avicoles, Jouy-en-Josas (S.-et-O.).

\section{PLAN DU MÉMOIRE}

\section{Introduction :}

a) Age au premier ouf et date d'éclosion.

b) Manifestation du génotype et date d'éclosion.

\section{Matériel :}
a) Animaux étudiés : races, origine.
b) Périodes analysées.
c) Sélection - mode de reproduction - consanguinité.
d) Organisation de l'élevage et données recueillies.

Méthodes :

\section{Résultats :}

a) Analyse de la variance à l'intérieur d'une génération.

b) Régression filles/mères pour le même père.

a) Distribution de la précocité dans les diverses années.

b) Composantes de la variance.

I $^{\circ}$ Composantes de la variance dans l'analyse entre groupes de sœurs et demi-sœurs.

$2^{\circ}$ Signification des variances.

$3^{\circ}$ Régression filles/mères.

$4^{\circ}$ Estimations de 1'héritabilité englobant tous les lots d'éclosion.

\section{Discussion des résultats :}

a) Interprétation génétique des différences entre animaux éclos tôt ou tard.

b) Interprétation de l'influence du milieu.

\section{Conclusions :}

a) Limites de cette étude.

b) Le problème de l'interaction hérédité $\times$ milieu.

c) Conséquences pratiques possibles.

d) Recherches ultérieures. 


\section{INTRODUCTION $\left(^{1}\right)$}

On a remarqué depuis longtemps que, chez certaines races comme la Bresse, les poulettes doivent, pour avoir une ponte d'hiver optimum, naître à une époque de l'année assez délimitée, tandis que pour d'autres, comme la Wyandotte, cela a moins d'importance.

D'une façon générale, il serait d'un grand intérêt pratique d'obtenir par sélection des animaux dont les caractères de ponte dépendent aussi peu que possible de la date d'éclosion.

Pour ce faire, il serait nécessaire de mettre en évidence l'existence de gènes de " résistance " ou de "sensibilité " aux différences de conditions de milieu provoquées par une date de naissance différente. Tel est l'objet de la présente étude, relativement à la précocité sexuelle.

Nous résumerons la littérature relative à l'influence de la date d'éclosion, d'une part sur l'âge au premier œuf, d'autre part sur la manifestation du génotype relatif à cette caractéristique.

\section{a) Age au premier cuf et date d'éclosion}

On sait depuis longtemps que l'âge de la ponte du premier ouf est conditionné par de nombreux facteurs du milieu parmi lesquels la date d'éclosion joue un rôle important.

JULL (I3) et HUTT (II) résument les travaux sur ce sujet. En particulier UPP et THOMPSON (22) remarquent que les poulettes écloses pendant la deuxième moitié de l'année sont plus précoces que les autres ; BYERLY et KNox (2) indiquent que 1'âge moyen au premier œuf augmente progressivement chez les animaux éclos après le 2I mars. C'est ce que constatent également TAYLOR et LERNER (2r), JEFFREY et PlatT (I2) et d'autres, cités par les mêmes auteurs.

Selon Greanwood et Blyth (6), la distribution de fréquences de 1'âge au premier œuf varie suivant la date d'éclosion, comme le montrent les distributions schématiques représentées ci-dessous :

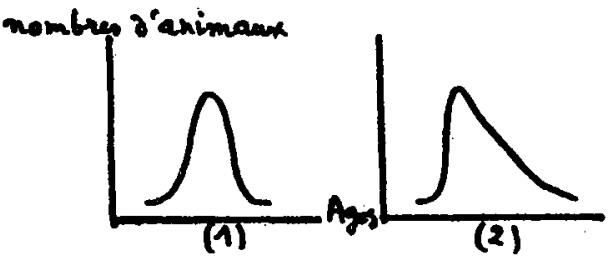

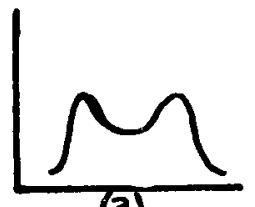

(3)

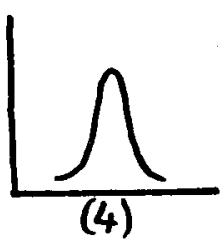

Les distributions du type (I) sont caractéristiques d'animaux nés tôt en saison, en février par exemple. L'âge moyen est faible, la distribution est peu étalée et symétrique.

(I) Ce travail a porté, pour une grande partie, sur les données de la S. A. R. G. A. S. (Station Avicole de Recherches Génétiques Appliquées à la Sélection) communiquées par le Dr L. P. Cochez, que nous remercions également pour ses remarques et ses critiques. 
Des animaux nés plus tard donnent des distributions du type (2), rendues dissymétriques par une certaine proportion d'animaux à maturité tardive, d'où un âge moyen et un étalement de la distribution plus grands.

Si l'éclosion a lieu encore plus tard (mai ou juin) on peut obtenir des distributions à plusieurs modes (type 3 ) et finalement un retour, en (4), à une distribution comme en (I).

Ces résultats conduisent à l'hypothèse d'une influence retardatrice de certaines conditions saisonnières, s'exerçant de plus en plus sur des animaux nés plus tard, puis s'estompant chez les animaux nés en fin de saison, qui retrouvent des conditions favorables à une bonne précocité.

Sous une latitude moins élevée, à Porto-Rico, GonZalez-ChAPEL et ROJAs-DAPORTA observent des phénomènes apparemment comparables (5).

\section{b) Manifestation du génotype et date d'éclosion}

C'est plus récemment qu'on a commencé à étudier l'influence possible de la date de naissance, non plus seulement sur l'âge au premier œuf, mais sur la manifestation des génotypes relatifs à cette mesure. GREENWOOD et BLYTH (6) suggèrent que les différences entre génotypes ne doivent atteindre leur expression maximum que quand les animaux approchent de leur maturité à l'automne, et que l'action des gènes de précocité pourrait s'exprimer en divers degrés de résistance à des conditions défavorables.

OSBORNE (I9) montre que, sur des Leghorns dorées consanguines à Edimbourg, le classement des diverses familles pour la précocité sexuelle n'est pas le même suivant que l'on considère les poulettes de ces familles nées tôt ou tard en saison. En d'autres termes, il y a une "interaction " significative entre génotype et date d'éclosion. L'auteur suggère que les entrées en ponte tardives de certaines poules des dernières couvées correspondent au fait que ces poules sont sensibles à la diminution rapide de durée du jour que l'on observe en automne. L'influence saisonnière est d'autre part suggérée par un ralentissement moyen de la croissance de la crête des poulettes, synchrone de la cessation de ponte des poules de plus d'un an.

ABplanalp (I) étudie les interactions possibles entre génotype et date d'éclosion en faisant l'analyse des paramètres génétiques sur un nombre croissant de couvées successives. Il trouve l'interaction la plus forte pour l'âge au premier œuf.

D'après Skalier et Sheldon (20), l'importance des interactions entre génotype et date d'éclosion pour l'âge au premier ouf varie suivant la souche ou la race étudiée. Ils mentionnent que, sur leurs animaux, aucun éclairement artiflciel n'était pratiqué, alors que le troupeau d'Édim- 
bourg recevait en automne un éclairage additionnel le matin, ce qui a pu amoindrir l'effet propre des conditions saisonnières.

Dans plusieurs cas, il s'est donc'avéré qu'une éclosion plus tardive ne se bornait pas à influer uniformément sur tous les génotypes, en retardant, par exemple, les entrées en ponte de la même durée chez tous les animaux. Au contraire, il y avait une action différentielle sur divers génotypes, certains étant plus " retardés " que d'autres. On peut parleı d'une " sensibilité " ou d'une "résistance " génotypique à des conditions saisonnières subies surtout par les dernières couvées à certains stades de leur maturation, et cette "résistance " n'a l'occasion de s'exprimer pleinement que chez ces dernières couvées.

I1 est malaisé dans de tels cas de juger de façon équivalente, en vue de leur sélection comme reproductrices, des poulettes nées à des dates sensiblement différentes. En particulier, une correction globale pour éliminer les différences entre les moyennes de précocité des diverses couvées est alors insuffisante. En outre, les meilleurs génotypes choisis dans un milieu (par exemple le milieu " naissance tôt en saison ") ne donnent pas obligatoirement les meilleures performances dans l'autre milieu.

Dans l'étude présente, nous ne pouvions vérifier directement si le classement des diverses familles pour l'âge au premier œuf était modifié par une date d'éclosion différente. La plupart des générations étudiées comportaient en effet deux pères successifs. Mais nous avons cherché à déterminer, pour deux périodes d'éclosion consécutives, la part respective de la variance due au milieu et de la variance génétique, et plus précisément de la variance génétique "additive ", suivant les méthodes d'analyse résumées par LERNER (I6).

Il est intéressant de savoir si la part de variance génotypique de l'âge au premier cuf varie, autrement dit si son héritabilité diffère, suivant la date d'éclosion. Celle-ci influera alors sur la rapidité du progrès obtenu par sélection, et sur le choix à faire entre sélection individuelle ou familiale.

\section{MATÉRIEL}

\section{a) Animaux étudiés : Races, origine}

Les recherches ont porté sur deux troupeaux. L'un, à la Station de Jouy-en-Josas, était composé de Bresse, de Gâtinaises, et de quelques animaux de croisement. Il s'agissait de souches plutôt légères, de ponte moyenne, à variations saisonnières notables. Ce troupeau avait été formé en I95 I à partir d'animaux de trois provenances différentes pour chaque race. Deux générations ont été étudiées, nées aux printemps I952 et I953. 
Le deuxième troupeau était constitué de Wyandottes à crête en rose, élevées près de Bordeaux (S.A.R.G.A.S.) ( ${ }^{1}$ ), sélectionnées de longue date pour une ponte forte et régulière. Cinq générations ont été suivies, nées aux printemps $1948,49,50,5$ I et 54 .

\section{b) Périodes analysées}

Cherchant à mettre en évidence l'influence de la date d'éclosion, nous avons choisi (sauf en 1954 dans le troupeau S.A.R.G.A.S.) les générations où deux pères successifs étaient accouplés aux mêmes poules. Après 1a mise en parquets du deuxième père, les œufs cessaient pendant I4 jours d'être incubés. Ceci permettait d'avoir une saison d'éclosion pédigree d'une durée totale de 8 à 9 semaines.

La descendance de chaque série de pères comportait 3 ou 4 lots éclos à intervalles d'une semaine. Les deux séries, correspondant à deux périodes d'éclosion successives, ont été traitées séparément du point de vue de l'analyse statistique. Dans le cas où il $\mathrm{y}$ avait un seul père, une subdivision en deux séries a été faite également, comprenant les premières et les dernières couvées.

Le tableau IV (p. r8o) donne les dates d'éclosion correspondant à chacune des deux séries étudiées pour chaque génération.

Le tableau I donne les nombres de pères, mères et enfants dans chaque série (désignée par $\mathrm{A}$ ou $\mathrm{B}$ ).

TABLEAU I

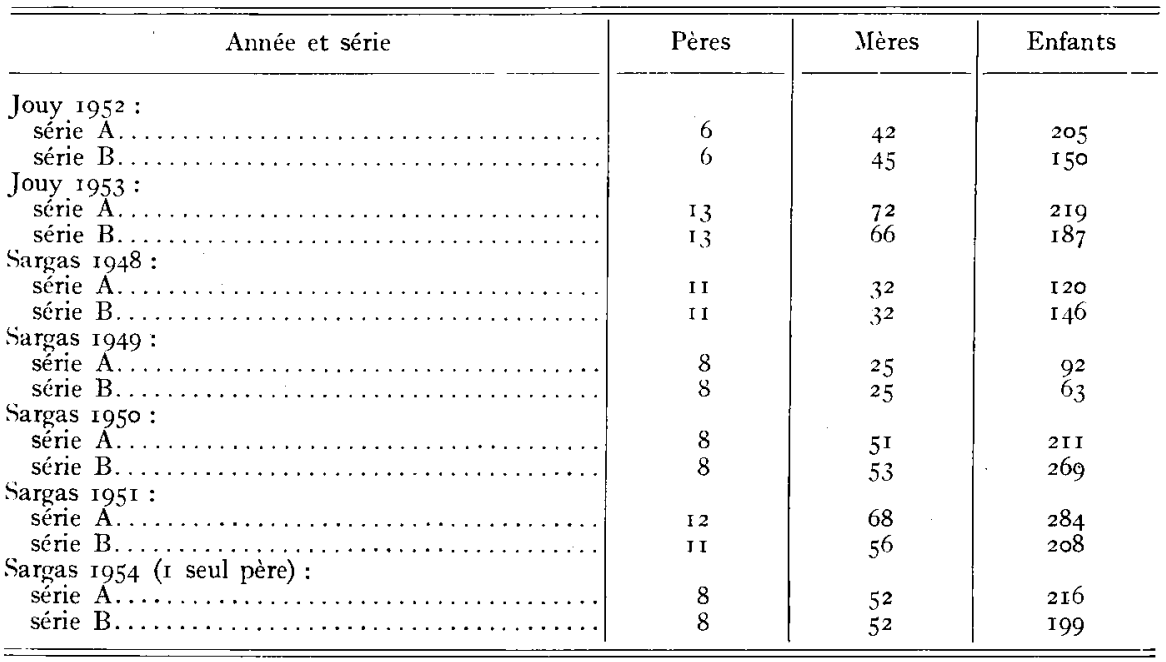

Le tableau II donne le détail des races ou croisements entrant dans l'analyse pour le troupeau de Jouy.

(I) Cf. page I74. 
TABLEAU II

\begin{tabular}{|c|c|c|c|}
\hline Année, série et race ou croisement & Pères & Mères & Enfants \\
\hline $\begin{array}{c}\text { Jouy I952, série A : } \\
\text { Bresse } \ldots \ldots \ldots \ldots \ldots\end{array}$ & & & \\
\hline Gâtinaise............. & $\begin{array}{c}3 \\
3\end{array}$ & $\begin{array}{l}21 \\
21\end{array}$ & 104 \\
\hline Jouy $195^{2}$, série $B$ : & & & \\
\hline $\begin{array}{l}\text { Bresse } \ldots \ldots \ldots \\
\text { Gâtinaise }\end{array}$ & 3 & 27 & 95 \\
\hline $\begin{array}{l}\text { Gâtinaise.......... } \\
\text { Jouy r953, série A: }\end{array}$ & 3 & 18 & 55 \\
\hline Bresse ............ & 6 & 28 & 78 \\
\hline Gâtinaise. & 6 & $3^{6}$ & I 10 \\
\hline FI ( $O$ Gâtinais $x$ ? Sussex) & I & 8 & $3 \mathrm{I}$ \\
\hline $\begin{array}{l}\text { Jouy I953, série } B \text { : } \\
\text { Bresse } \ldots \ldots \ldots \ldots\end{array}$ & 6 & 27 & 69 \\
\hline Gâtinaise. & 6 & 32 & 86 \\
\hline $\mathrm{F}_{\mathrm{I}}$ ( $O$ Gâtinais $\times$ Sussex $)$ & I & 7 & $3^{2}$ \\
\hline
\end{tabular}

\section{c) Sélection, mode de reproduction, consanguinité}

Les deux troupeaux sont sélectionnés sur un ensemble de caractères incluant des mesures de la croissance et de la ponte.

Le mode d'accouplement des reproducteurs a varié un peu suivant les générations. Certaines années, des poules accouplées au même coq étaient sœurs ou demi-sœurs entre elles, alors que d'autres années ceci a été systématiquement évité. Dans le premier cas, les enfants d'un même père pourraient se ressembler un peu plus que dans le second, puisque certaines de leurs mères sont parentes entre elles. Cependant, ceci ne parait pas avoir joué un rôle important.

Toute consanguinité systématique a été évitée. Il n'existe dans les deux troupeaux que la consanguinité pouvant résulter de 1'élevage en troupeau "fermé " pendant un certain nombre d'années.

Le fait principal est que la sélection, le mode d'accouplement des reproducteurs et le taux de consanguinité ont été, à chaque génération et autant que cela a pu être contrôlé, identiques pour la première et la deuxième série d'éclosions. Notre but essentiel ici a été la comparaison des paramètres génétiques entre les deux séries, non la détermination de la valeur absolue de ces paramètres. Cette dernière nécessiterait une estimation du taux moyen de consanguinité, qui n'a pas été faite ici. D'autre part, à Jouy, les analyses ont porté sur plusieurs races ou croisements groupés, ce qui serait à considérer si l'on voulait rapprocher les estimations correspondantes de l'héritabilité, prises en valeur absolue, d'autres estimations faites sur une race unique.

\section{d) Organisation de l'élevage et données recueillies}

Toutes les conditions contrôlées de l'élevage, alimentation, traitements et manipulations éventuels, ont été, autant qu'il était possible, 
identiques pour les-premiers et les derniers lots. Des variations entre années ont eu lieu, particulièrement à Jouy pour l'alimentation.

Aucune lumière artificielle n'a été donnée, à aucun moment, dans les différentes années analysées.

La mortalité, d'une façon générale, n'a pas été très forte jusqu'au $3 \mathrm{I}$ - I2, pour les années étudiées, et n'a pas notablement différé pour lés divers lots. Aucune autre élimination n'est faite en cours d'élevage que celle des malades. Seules font partie de l'analyse les poulettes ayant survécu jusqu'en janvier.

Les diverses familles sont mélangées dans les mêmes locaux.

Les reproducteurs sont, pour la majorité, des animaux de moins d'un an.

\section{MÉTHODES}

Deux méthodes ont été employées pour estimer les héritabilités :

\section{a) Analyse de la variance à l'intérieur d'une génération}

La méthode suivie ici a été exposée par WINSOR et CLARKE (27), et l'application au calcul de l'héritabilité a été détaillée par KING et HENDERSON (I4).

Elle permet, à partir des estimations de la variance "entre pères", " entre mères pour le même père " et " intra-mères ", d'évaluer les composantes de la variance dues à l'effet du père, à l'effet de la mère et "résiduelle ". La décomposition hiérarchique des écarts permet de tenir compte du fait que les effectifs des diverses familles de scurs ou de demisœurs sont inégaux.

Outre les estimations des composantes de la variance, nous avons déterminé dans tous les cas le rapport de la variance "entre pères " et " entre mères intra-pères " à la variance "résiduelle " dans le but de voir si les différences entre familles de pères ou de mères, étaient, dans leur ensemble, significatives (VESSEREAU, 23).

\section{b) Régression filles/mères pour le même père}

Cette méthode est décrite en particulier par WhatLEy (26) et KorkMAN (I5). Employée seulement ici comme recoupement de la précédente, elle n'a porté que sur la génération 1953 de Jouy, comparée aux mères nées en 1952. D'une façon analogue à la première méthode, la régression a été calculée à part suivant que la mère appartenait aux premiers ou aux derniers lots, ainsi que les enfants. Il en résulte quatre analyses différentes pour lesquelles le tableau III donne les nombres de mères et d'enfants (A désignant la première série d'éclosions et $\mathrm{B}$ la deuxième, dans l'une et l'autre génération). 
TABLEAU III

Effectifs pour l'analyse de la régression filles/mères (Jouy, I952-I953)

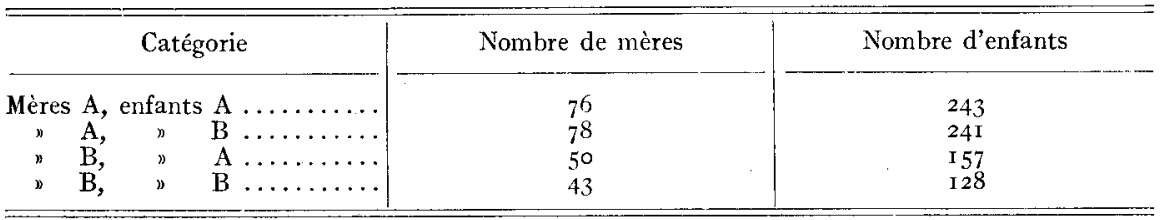

\section{RÉSULTATS}

\section{a) Distribution de la précocité dans les diverses années étudiées}

Le tableau IV donne, en regard des dates extrêmes de naissance des diverses séries, l'âge moyen de l'entrée en ponte et sa variance.

TABleau IV

\begin{tabular}{|c|c|c|c|}
\hline Année et série & Dates d'éclosion & $\begin{array}{l}\text { Age moyen au } \\
\text { I er ceuf (jours) }\end{array} \mid$ & Variance totale \\
\hline $\begin{array}{l}\text { Jouy } \\
\text { Série } \AA\end{array}$ & & 100 & I 875 \\
\hline Série $B \ldots \ldots \ldots \ldots \ldots \ldots$ & $9 / 4$ au $23 / 4$ & $\begin{array}{l}190 \\
213\end{array}$ & $\begin{array}{r}1075 \\
947\end{array}$ \\
\hline Jouy I953 : & & & \\
\hline Série $\mathrm{A} \ldots \ldots \ldots \ldots \ldots \ldots \ldots \ldots$ & $6 / 3$ au $20 / 3$ & 168 & 600 \\
\hline $\begin{array}{l}\text { Série B } \ldots \ldots \ldots \ldots \ldots \ldots \ldots \\
\text { Sargas I } 948 \text { : }\end{array}$ & II $/ 4$ au $25 / 4$ & 187 & I 427 \\
\hline $\begin{array}{l}\text { Série A } \ldots \ldots \ldots \ldots \ldots \ldots \ldots \ldots \ldots \ldots \ldots \ldots \ldots \ldots \ldots \\
\text { Série B } \ldots \ldots \ldots \ldots \ldots \ldots \ldots\end{array}$ & $\begin{array}{l}\text { I } 6 / 3 \text { au } 6 / 4 \\
20 / 4 \text { au } \\
\text { I } 1 / 5\end{array}$ & $\begin{array}{l}\text { I } 88 \\
20 I\end{array}$ & $\begin{array}{l}719 \\
383\end{array}$ \\
\hline Sargas I $949:$ & $20 / 4$ at $11 / 5$ & & \\
\hline Série A ... & $9 / 3$ au $23 / 3$ & 170 & 379 \\
\hline $\begin{array}{l}\text { Série B } \ldots . \cdots \cdots \cdots \\
\text { Sargas I950: }\end{array}$ & $20 / 4$ au $4 / 5$ & 172 & 473 \\
\hline Série A.... & $22 / 2$ au $8 / 3$ & 176 & $\mathbf{I} 69$ \\
\hline $\begin{array}{l}\text { Série B } \ldots \ldots \ldots \ldots \\
\text { Sargas I951 : }\end{array}$ & $29 / 3$ au $I 2 / 4$ & $18 \mathrm{I}$ & 257 \\
\hline Série A .... & $22 / 2$ au $8 / 3$ & 180 & $29 \mathrm{I}$ \\
\hline Série $B \ldots \ldots$ & $29 / 3$ au I $2 / 4$ & 190 & $65^{2}$ \\
\hline $\begin{array}{l}\text { Sargas I954 : } \\
\quad \text { Série } A \ldots \ldots \ldots \ldots \ldots \ldots \ldots \ldots \ldots \ldots \ldots \ldots \ldots \\
\text { Série } B \ldots \ldots \ldots \ldots \ldots \ldots\end{array}$ & $\begin{array}{l}22 / 2 \text { au I } 5 / 3 \\
22 / 3 \text { au I } 2 / 4\end{array}$ & $\begin{array}{l}\text { I87 } \\
\text { I } 86\end{array}$ & $\begin{array}{l}492 \\
667\end{array}$ \\
\hline
\end{tabular}

Le tableau $\mathrm{V}$ fait apparaitre les distributions de fréquences des dates d'entrée en ponte (en nombre de jours avant la fin de l'année).

I a médiane est indiquée par une flèche, et les entrées en ponte ultérieures au 3I/I2 ont été marquées sur la colonne d'abscisse zéro.

L'examen des graphiques confirme sur nos données la tendance des distributions, indiquée par GREENwOOD et BLYTH (6), à devenir plus étalées et plus obliques dans lesderniers lots, dont 1'entrée en ponte se fait plus tard en saison. Ceci est visible en I953 (Jouy) et I949, I950, I95 I (S.A.R.G.A.S.) où, grosso modo, les premiers lots entrent en ponte en août-septembre et les derniers I mois ou I mois I/2 plus tard. 


\section{Tableau V}
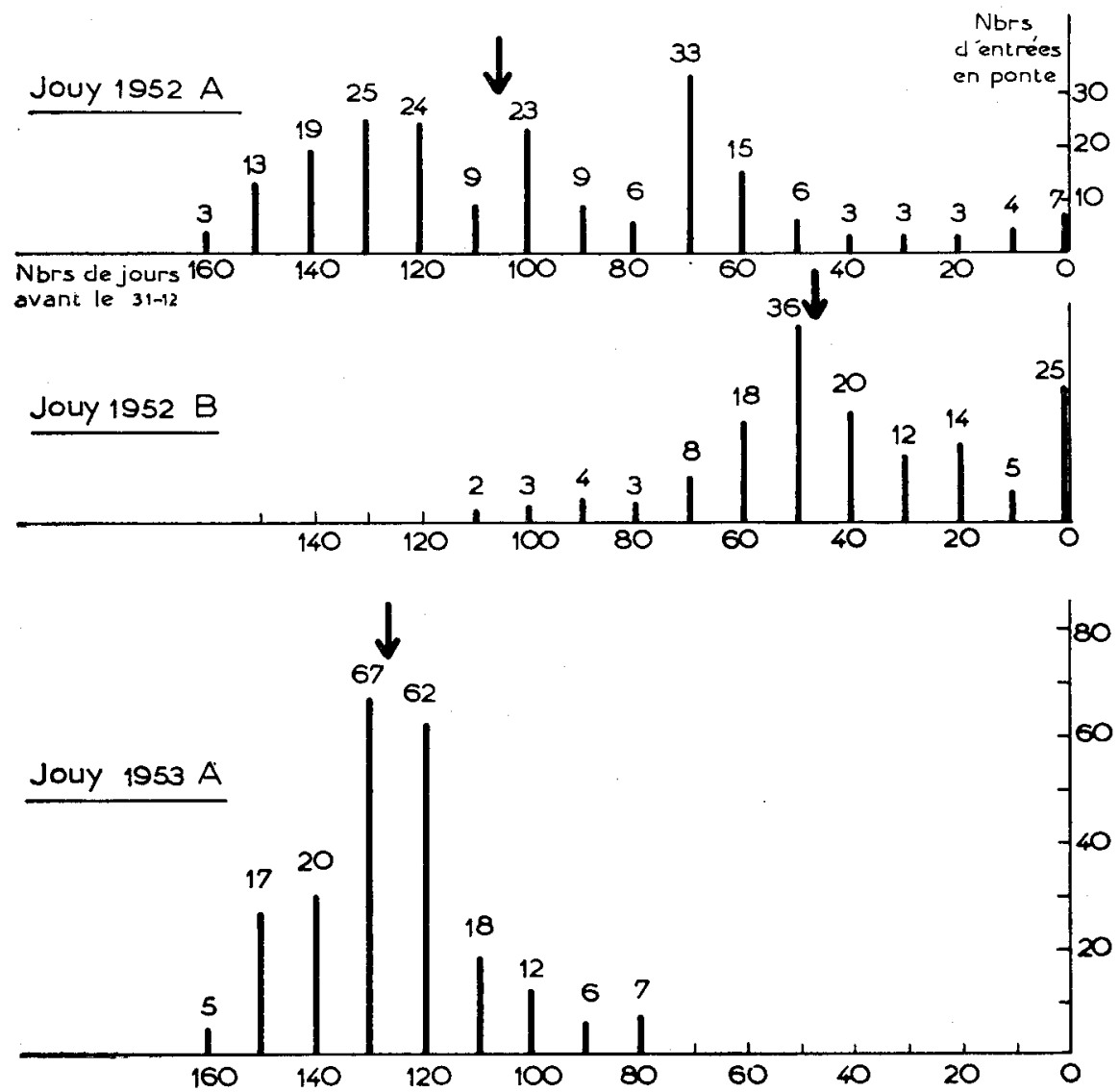

Jouy $1953 B$

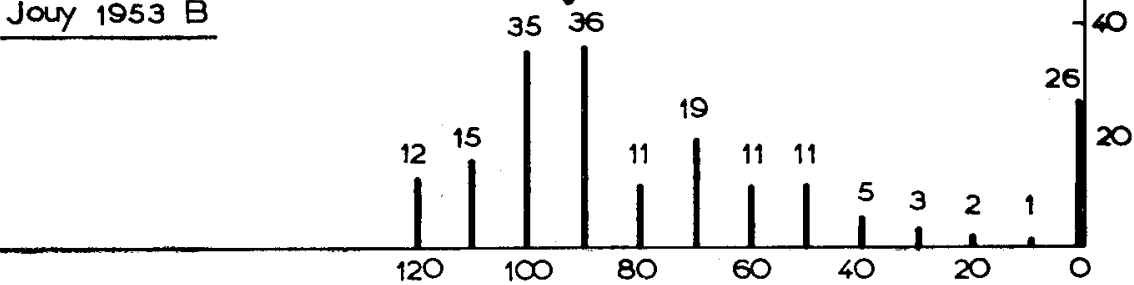




\section{TablefaU V (suite)}
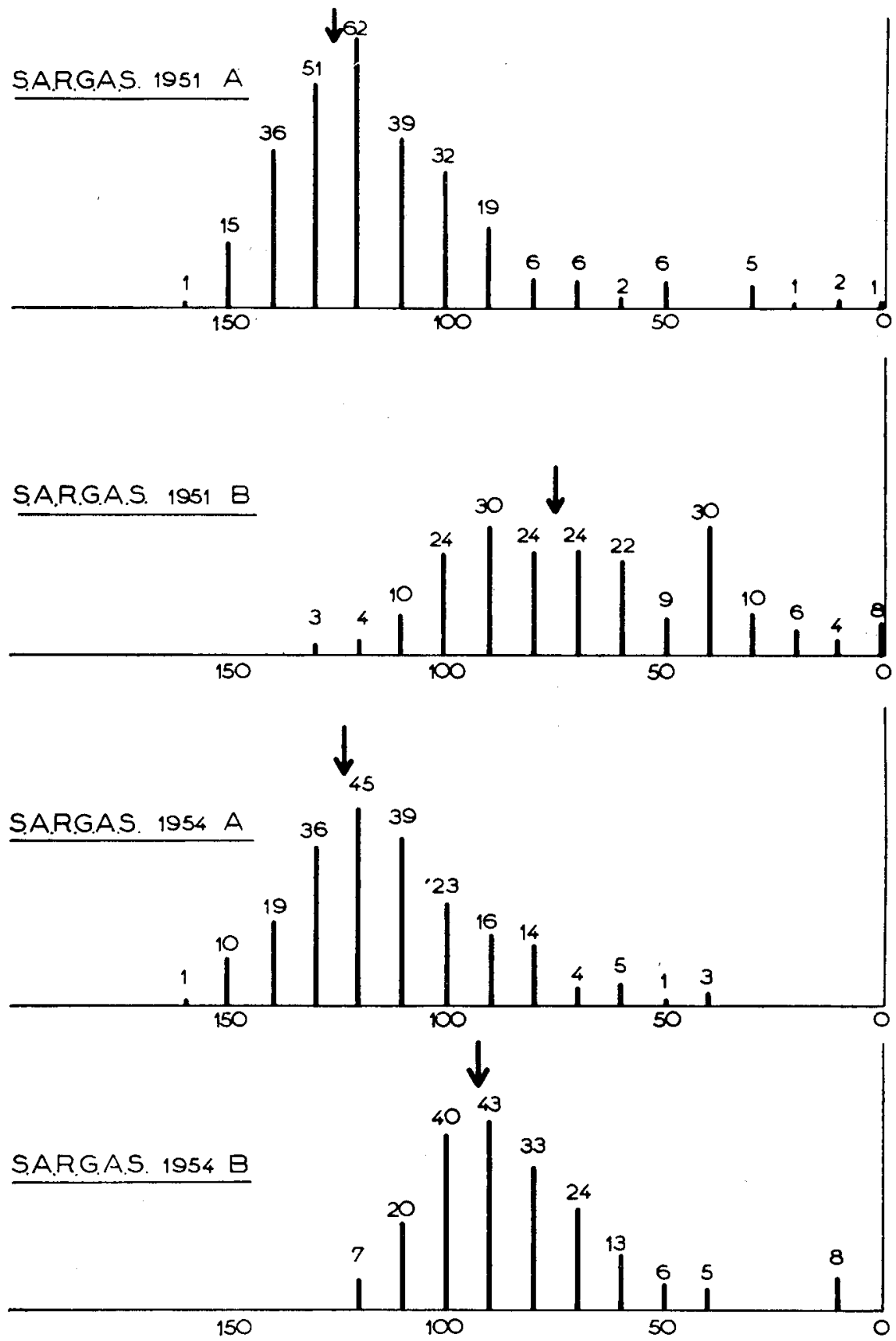
TABLEAU V (suite)

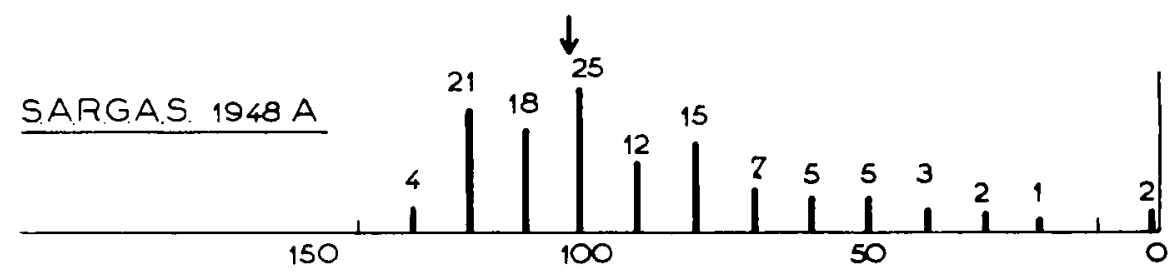

SARGAS $1948 \quad B$
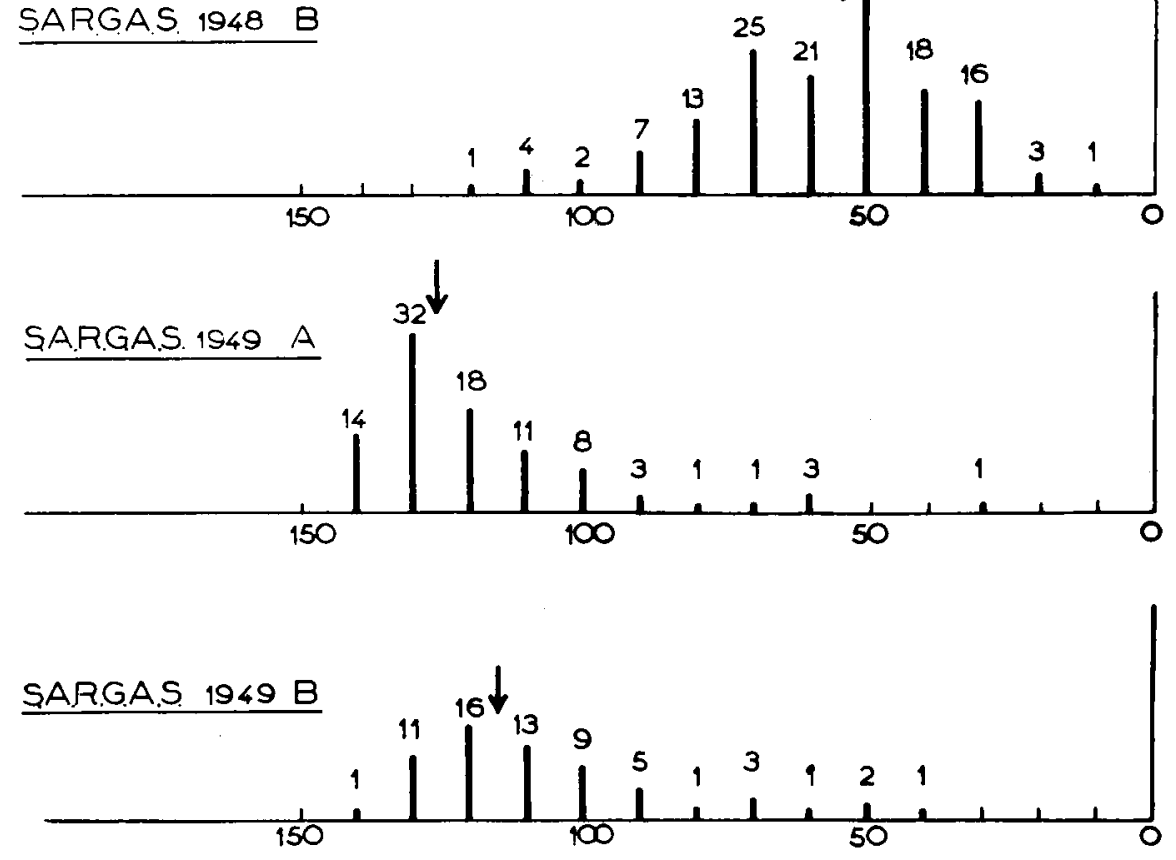

Les années I952 (Jouy) et I948 (S.A.R.G.A.S.) confirment de même que, quand l'entrée en ponte des derniers lots d'éclosion est encore plus tardive (maximum en novembre ou décembre,) la distribution redevient normale et moins étalée.

Ces faits sont reflétés par la comparaison des âges moyens au premier ouf et des variances dans le tableau IV. Ils font apparaittre vraisemblable la présence en automne d'une époque où les entrées en ponte sont " retardées ». L'allure des distributions paraitt liée à la date des entrées en ponte plus directement qu'à la date d'éclosion.

Le tableau VI montre que la plupart des distributions s'écartent de la normalité d'une façon significative (VESSEREAU, 23, page 74). 
TABLEAU V (fin).

\section{SARGA.S. 1950 A}
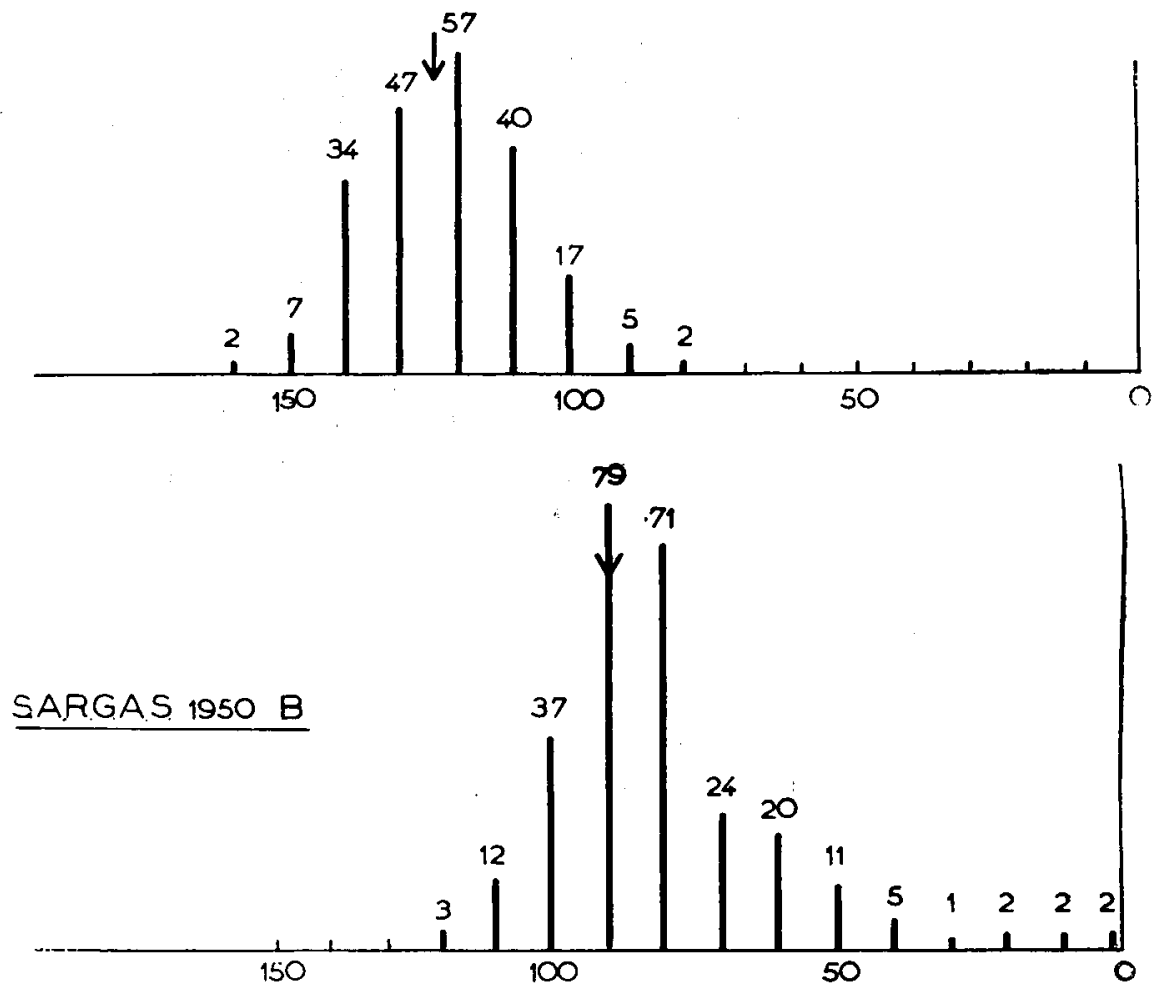

\section{TABLEAU VI}

\begin{tabular}{l|c|c|c|c}
\hline \hline & $\begin{array}{c}\text { Jouy } \\
\text { I952 A }\end{array}$ & $\begin{array}{c}\text { Jouy } \\
\text { I952 B }\end{array}$ & $\begin{array}{c}\text { Jouy } \\
\text { I953 A }\end{array}$ & $\begin{array}{c}\text { Jouy } \\
\text { I953 B }\end{array}$ \\
\hline Distribution..... & S.r $\%$ & S. I $\%$ & S.I $\%$ & S. I \% \\
\hline
\end{tabular}

S. A. R. G. A. S.

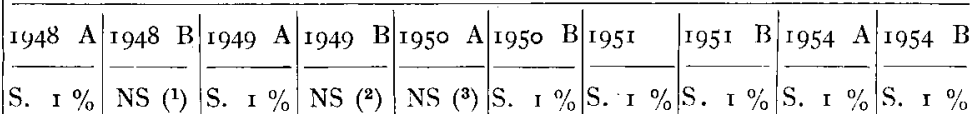

(') $0,1<\mathrm{p}<0,2 .-\left({ }^{2}\right) \quad 0,1$ 样 $\mathrm{p},-\left({ }^{3}\right) \quad 0,5<\mathrm{p}<0,7$ 


\section{b) Composantes de la variance}

\section{I. - Analyse entre groupes de sœurs et demi-sœurs}

Le tableau VII rassemble les estimations des composantes de la variance et de l'héritabilité, d'après la composante "mère " et d'après la composante "père ".

Les notations employées sont empruntées à KING et HENDERSON (I4) et ont la signification suivante :

$s^{2}$ : composante " résiduelle " de la variance, dûe aux écarts entre sœurs ; contient la plus grande partie de la variance dûe au milieu, plus une part de la variance génotypique.

$s_{d}^{2}$ : composante " mère " de la variance, dûe à l'apport commun de la mère à tous ses enfants.

$s_{s}^{2}$ : composante " père " de la variance, dûe à l'apport commun du père à tous ses enfants.

$s_{d}^{2}$ et $s^{2}$ contiennent chacun une fraction de la variance génotypique.

On sait (LERNER, I6, page I20) que, si l'accouplement des reproducteurs a lieu " au hasard " et s'il n'y a pas de corrections à faire pour la consanguinité, on peut calculer les deux estimations indépendantes suivantes de l'héritabilité $\left({ }^{1}\right)$ :

$$
h_{d}^{2}=\frac{4 s_{d}^{2}}{s_{e}^{2}+s_{d}^{2}+s_{s}^{2}} \quad \text { et } \quad h_{s}^{2}=\frac{4 s_{s}^{2}}{s_{e}^{2}+s_{d}^{2}+s_{s}^{2}}
$$

d'où les deux dernières colonnes du tableau.

Pour simplifier, nous n'avons pas mentionné sur ce tableau les sommes de carrés ayant servi à calculer les composantes de la variance, avec les degrés de liberté correspondants.

\section{2. - Signification des variances}

Pour chaque série de chaque génération, nous avons comparé les variances " entre mères intra-pères " et " entre pères " à la variance " résiduelle $"$.

Le tableau VIII montre le degré de signification que l'on pourrait accorder à la différence de ces variances d'avec la variance résiduelle, si l'on pouvait partout utiliser les tests de signification ordinaires. Mais ces tests sont définis pour des distributions voisines de la normale, ce qui n'est pas le cas ici en général (cf. tableau VI).

(1) $s_{e}^{2}, s_{d}$ et $s_{s}^{2}$ sont souvent appelés $\mathrm{Q}, \mathrm{D}$ et $\mathrm{S}$ par les auteurs anglo-saxons. 
TABI,EAU VII ( $\left.{ }^{\mathbf{l}}\right)$

\begin{tabular}{|c|c|c|c|c|c|}
\hline Année et série & $\mathrm{s}_{\rho}^{2}$ & $s_{d}^{2}$ & $\mathrm{~s}_{8}^{2}$ & $\mathrm{~h}_{d}^{s}$ & $\mathrm{~h}_{s}^{2}$ \\
\hline \multirow{2}{*}{\multicolumn{6}{|c|}{$\begin{array}{l}\text { Jouy } 1952 ; \\
\text { A } \ldots \ldots \ldots \ldots \ldots \ldots\end{array}$}} \\
\hline & I $33^{8}$ & $33^{8}$ & I99 & 0,72 & 0,42 \\
\hline B $\ldots \ldots \ldots \ldots \ldots \ldots \ldots$ & $79 \mathrm{I}$ & $(-65)$ & $22 \mathrm{I}$ & $(-0,24)$ & 0,82 \\
\hline A $\ldots \ldots \ldots \ldots \ldots \ldots \ldots \ldots \ldots \ldots \ldots$ & 460 & I 26 & I4 & 0,84 & 0,09 \\
\hline$B \ldots \ldots \ldots \ldots \ldots \ldots$ & I 098 & I6 & $3 \mathbf{I} 3$ & 0,04 & 0,87 \\
\hline \multicolumn{6}{|l|}{ Sargas I948: } \\
\hline$A \ldots \ldots \ldots \ldots \ldots \ldots$ & $5^{80}$ & $8 \mathbf{I}$ & 68 & 0,44 & 0,37 \\
\hline \multicolumn{5}{|l|}{ Sargas I949: } & 0,18 \\
\hline$A \ldots \ldots \ldots \ldots \ldots \ldots$ & 328 & 47 & 8 & 0,49 & 0,08 \\
\hline & \multicolumn{4}{|c|}{ Sargas I950: } & 2,23 \\
\hline A........... & I30 & 23 & I6 & 0,54 & $0,3^{8}$ \\
\hline \multicolumn{5}{|l|}{ Sargas 195 I : } & 0,12 \\
\hline A $\ldots \ldots \ldots \ldots \ldots \ldots \ldots$ & $25 \mathrm{I}$ & I8 & 22 & 0,24 & $0,3^{\circ}$ \\
\hline$\underset{\text { Sargas ro54: }}{B} \cdots \cdots \cdots \cdots$ & 559 & $(-30)$ & 123 & $(-0,17)$ & 0,69 \\
\hline A $\ldots \ldots \ldots \ldots \ldots \ldots \ldots$ & 403 & 26 & 63 & $0,2 \mathrm{I}$ & $0,5^{2}$ \\
\hline$B \ldots \ldots \ldots$ & $55^{2}$ & $4^{8}$ & 67 & 0,29 & 0,40 \\
\hline
\end{tabular}

TABLEAU VIII

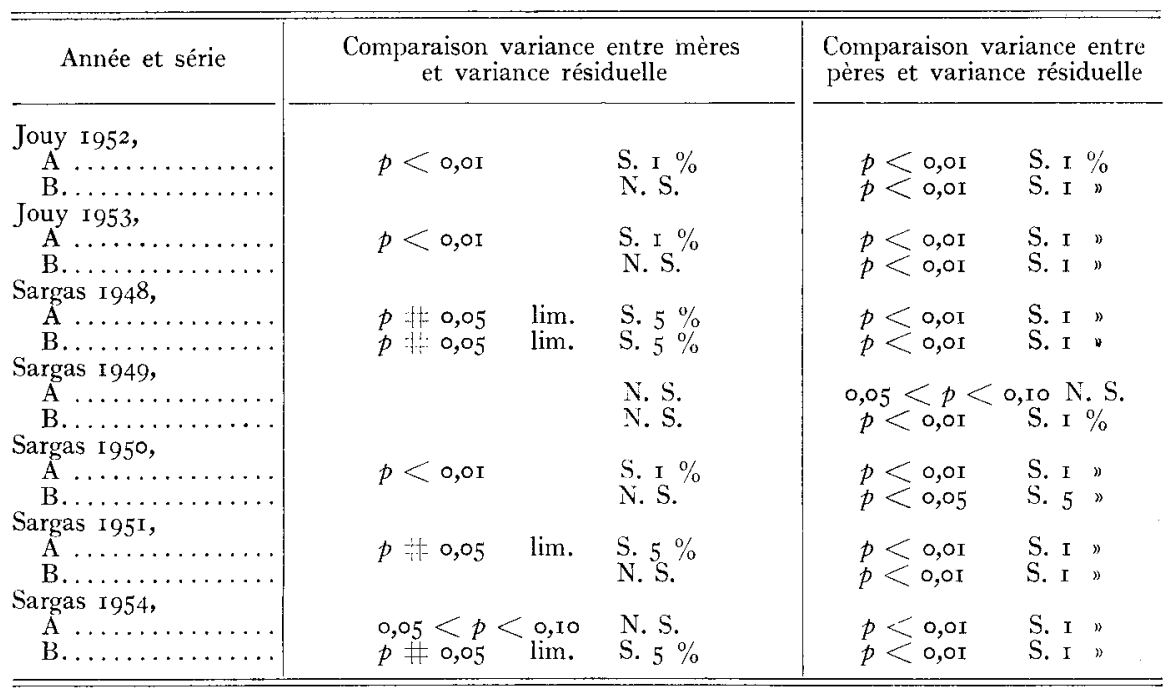

\section{3. - Régression filles/mères}

I,e tableau IX donne les coefficients de la régression filles/mères pour le même père, pour les poulettes de Jouy I953, comparées à leurs mères nées en I952, suivant la date d'éclosion des unes et des autres.

Nous avons estimé le taux d'héritabilité $h^{2}$ comme le double dt coefficient de régression $b$ (WHATLEY, 26).

(1) L'estimation des composantes "mère " et " père " de la variance fait intervenir des différences. Ceci explique que, sur des échantillons limités, on obtienne parfois des composantes " mère " négatives, qui en fait ne diffèrent pas significativement de zéro. Elles ont été mises entre parenthèses, ainsi que les valeurs $h_{d}^{2}$ correspondantes. 
TABLEAU IX

\begin{tabular}{|c|c|c|}
\hline Séries & $\begin{array}{l}\text { Caefficient de } \\
\text { régression }\end{array}$ & $\mathrm{h}^{2}$ \\
\hline 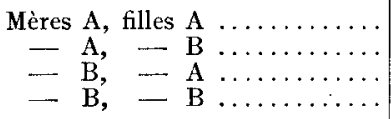 & $\begin{array}{l}+0,33 \\
\pm 0,09 \\
+0,15 \\
0,09\end{array}$ & $\begin{array}{c}0,66 \\
(-0,18) \\
0,30 \\
-0,18)\end{array}$ \\
\hline
\end{tabular}

\section{4. - Estimations de l'héritabilité englobant tous les lots d'éclosion}

L'héritabilité a été aussi estimée sur l'ensemble des deux séries A et $B$ d'éclosions, pour les diverses années. Les résultats sont consignés dans le tableau $X$.

\section{TABLEAU $\mathrm{X}$}

\begin{tabular}{|c|c|c|c|c|c|}
\hline Année & $\mathrm{s}_{e}^{2}$ & $\mathrm{~s}_{d}^{2}$ & $\mathrm{~s}_{3}^{2}$ & $\mathrm{~h}_{d}^{2}$ & $\mathrm{~h}_{s}^{2}$ \\
\hline \multicolumn{6}{|l|}{ Jouy : } \\
\hline $195^{2}$ & II 24 & I47 & $33^{6}$ & 0,36 & 0,83 \\
\hline r953. & $74^{8}$ & $8 \mathrm{I}$ & 235 & $0,3^{\circ}$ & 0,88 \\
\hline \multicolumn{6}{|l|}{ Sargas : } \\
\hline I948 & 436 & 65 & 74 & 0,45 & $0,5^{\mathrm{I}}$ \\
\hline 1949 & 295 & II & I18 & 0,10 & $\mathrm{I}, \mathrm{II}$ \\
\hline $1950 \ldots \ldots$ & 196 & Io & I6 & 0,18 & 0,29 \\
\hline I95I & 378 & 0 & 7 & 0,00 & 0,70 \\
\hline $1954 \ldots$ & 463 & $4^{6}$ & 65 & 0,32 & 0,45 \\
\hline
\end{tabular}

\section{DISCUSSION DES RÉSULTATS}

\section{a) Interprétation génétique des difiérences entre animaux éclos tôt ou tard}

Les tableaux VII et VIII nous donnent les éléments de comparaison entre composantes de la variance et héritabilités chez les animaux éclos tôt ou tard.

Comme on sait, l'héritabilité est une estimation de la proportion de 1a variance génétique " additive " par rapport à la variance totale.

\section{I. - Composante mère}

En ce qui concerne la variance due à l'apport commun de la mère à ses enfants, et l'estimation correspondante de l'héritabilité, nous voyons que, dans cinq cas sur sept, cette estimation est plus grande pour les premiers lots, entrés en ponte plus tôt.

Quoique toutes les distributions de l'âge au premier œuf ne.puissent être considérées comme normales, le tableau VIII montre la mesure dans laquelle, si c'était le cas, on pourrait considérer comme significatives les différences entre mères dans leur ensemble. Nous voyons que la va- 
riance entre mères, pour les derniers lots, approcherait dans 2 'cas seulement du seuil 5 p. Ioo de signification, alors que la signification, pour les premiers lots, dépasserait trois fois le seuil I p. Ioo et atteindrait deux fois 5 p. IOO.

De plus, en I954, où 1'héritabilité estimée par la composante mère n'est pas plus grande pour les premiers lots, l'écart des dates d'éclosion entre série " $A$ " et série " $B$ " était amoindri par l'absence d’interruption dans les incubations.

Si, enfin, pour fixer les idées, nous faisons pour toutes les générations étudiées la moyenne des valeurs $h_{d}^{2}$, nous obtenons :

0,50 pour les premiers lots (série $\mathrm{A}$ )

et 0,03 pour les derniers (série $B$ ).

Pour les années I952 et I953 de Jouy, cette tendance est confirmée par l'examen des régressions filles/mères suivant la date d'éclosion.

Les différences des familles de sœurs (issues d'une même mère) entre elles sont donc mieux tranchées chez les lots entrés en ponte moins tard en saison. Cela peut logiquement provenir de deux causes : la première est la présence d'une plus grande proportion de variance génotypique et notamment de variance génotypique «additive » dans la variance totale.

La seconde est une ressemblance plus grande entre les sœurs qu'entre des individus non parents, causée par le milieu.

Fn ce qui concerne une influence commune du milieu extérieur sur les enfants d'une même mère, le fait que les diverses familles ont été mélangées dès la naissance dans les mêmes locaux rend son importance improbable. I1 reste la possibilité d'un effet maternel transmis par l'œuf. Les évaluations statistiques de l'action commune du milieu sur des sœurs, incluant cet effet maternel (HAzEL, et LAMOREUX, IO ; LERNER et CrUDEN, I7) ont fait conclure à son peu d'importance en ce qui concerne la précocité sexuelle. ONishi (I8) trouve une héritabilité par la " composante mère " notablement plus grande que celle obtenue par la " composante père »; il s'agit de lignées consanguines et de leurs croisements ; ceci pourrait s'expliquer, au moins en partie, par une interaction entre gènes de la mère et gènes du père.

En résumé, on ne peut ici exclure à priori 1'hypothèse d'un effet maternel sur la maturité sexuelle, beaucoup plus important chez des animaux éclos plus tôt. Cependant, on a rarement mis en évidence un tel effet.

D'autre part, la distribution des entrées en ponte semble liée aux conditions saisonnières précédant ou accompagnant l'arrivée des poulettes à maturité, plutôt qu'à des circonstances antérieures. Il est donc plus vraisemblable de penser que les différences génotypiques se manifestent davantage chez les poulettes entrant en ponte tôt en saison, du moins dans les limites de la présente étude. 


\section{2. - Composante père}

Pour la comparaison entre groupes d'enfants de pères différents, remarquons tout d'abord que les estimations d'héritabilité correspondantes $\left(h_{s}^{2}\right)$ comportent une certaine erreur d'échantillonnage, le nombre de pères étant d'une dizaine par série. Cependant, l'ensemble des données groupe près de 70 pères dans chaque série.

Cette réserve faite, nous voyons que, sur six générations, $h_{s}^{2}$ est supérieur à $h_{d}^{2}$ dans la série $\mathrm{B}$, ce qui suggère la présence d'une forte variabilité liée au sexe, alors que seule l'année 1954 fait apparaître dans la série A une valeur $h_{s}^{2}$ nettement supérieure à $h_{d}^{2}$.

Quant à la comparaison entre les estimations de $h_{s}^{2}$ dans les séries A et $\mathrm{B}$, elle montre que, dans l'ensemble, $h_{s}^{2}$ est plus grand en B, contrairement à ce qui se passe pour l'héritabilité estimée par la "composante mère ».

Pour fixer les idées, nous trouvons comme valeurs moyennes, dans la série $\mathrm{B}: h_{s}^{2}=0,76$ (ou $0,5 \mathrm{I}$ si l'on ne tient pas compte de la valeur anormalement élevée du troupeau S.A.R.G.A.S. I949, ne portant que sur 63 enfants, comme l'indique le tableau I) et $h_{d}^{2}=0,03$

alors que dans la série A ces valeurs sont respectivement :

$0,3 \mathrm{I}$ et 0,5 .

Nous observons donc en $B$ une variabilité liée au sexe importante, alors qu'en $\mathrm{A}$, pour permettre de conclure à l'existence d'une telle variabilité, il faudrait admettre qu'elle est masquée par un "effet maternel " grossissant considérablement la valeur $h_{d}^{2}\left({ }^{1}\right)$. (Nous avons vu que l'hypothèse de l'effet maternel ne semble pas très vraisemblable). L'existence de gènes liés au sexe et influant sur la maturité sexuelle des poulettes a été mentionnée plusieurs fois, notamment par Hays (9) sur des RhodesIsland, par WARREN (24), (25) sur des croisements Leghorn blanche $\times$ Rhode, par ONISHI (I8) sur des croisements entre lignées consanguines de Leghorns blanches.

Les résultats présents tendent à montrer de plus que la manifestation de tels gènes peut dépendre de la saison d'entrée en ponte ; leur action correspondrait à une résistance plus ou moins grande aux conditions de l'automne défavorables à une maturation sexuelle précoce.

\section{b) Interprétation de l'influence du milieu}

I'étude présente indique, sur les races et dans les conditions mentionnées, une manifestation différente des génotypes suivant la date d'éclosion. Elle rejoint, quoique par une autre voie, les conclusions d'Osborne (I9), d'ABplanal,p (I) et de SkalitirR et Sheldon (20).

(1) Ou par des interactions non-additives entre gènes, dont une part plus grande serait incluse dans $h_{d}^{2}$ que dans $h_{s}^{2}$. 
Les facteurs du milieu provoquant cette manifestation différente sont liés à la date de naissance. Ils peuvent donc provenir, soit de conditions saisonnières différentes, soit de conditions accidentelles d'élevage, d'alimentation ou autres, faisant qu'une couvée n'a pas été traitée exactement de la même façon qu'une autre. Cependant, le fait que nous nous soyons toujours efforcés de fournir les mêmes conditions contrôlées d'élevage quelle que soit la date d'éclosion et le sens toujours le même des différences entre lots éclos plus tôt et plus tard pour $\mathrm{h}_{a}^{2}$ militent en faveur d'une influence saisonnière.

Comme GreEnWOOD et Bryth (6) l'ont mentionné, la plus grande proportion de " retardataires " quand le gros des entrées en ponte a lieu vers le moment où les jours raccourcissent le plus vite, suggère la nature possible de cet effet saisonnier. L'influence de la durée de l'éclairement diurne sur la ponte est connue, et la même période de l'automne voit le maximum des arrêts de ponte des poulettes, et des mues chez les poules d'un an. Il reste que ceci est seulement une indication, et que par ailleurs d'autres conditions saisonnières peuvent aussi intervenir.

\section{CONCLUSIONS}

\section{a) Limites de cette étude}

Les faits établis et discutés précédemment ne sont évidemment prouvés que pour les races et plus précisément les souches en question et pour les conditions de milieu où elles se sont trouvées, conditions générales d'élevage et période d'éclosion $\left(^{1}\right)$. Cependant, il parait intéressant que deux troupeaux de races différentes, élevés en des localités éloignées, manifestent des tendances analogues sur plusieurs générations chacun.

\section{b) Le prohlème de l'interaction hérédité $\times$ milieı}

Les résultats obtenus peuvent se rattacher au problème général des interactions entre hérédité et milieu, classées qualitativement par HALDANE (7). L,e génotype pour la maturité sexuelle ne se manifeste pas toujours de la même façon à une époque ou à une autre de l'année, comme l'ont déjà fait ressortir OsBorne, SkALLER et SHELdon.

GREENWOOD et BLYTH, déjà cités, pensent que l'expression des différences génotypiques devrait être maximum à l'automne, où les animaux pourraient manifester leur résistance naturelle aux conditions défavorables du milieu. I1 est intéressant de constater que, sur nos animaux, ceci paraît vérifié quant à des gènes liés au sexe. Par contre, en ce qui concerne la variance génotypique estimée d'après la "composante mère ", c'est l'inverse qui se passe; or, cette estimation est une indication de la

(1) Les termes " nés tôt " ou « tard " au printemps seraient à analyser plus avant, compte tenu, peut-être, des conditions de milieu particulières à chaque année et influant sur les entrées en ponte. 
variabilité "additive " provenant des gènes autosomaux. Si l'on admet que les conditions extérieures correspondant à une entrée en ponte plus tard dans l'automne constituent un milieu "défavorable " à une maturité précoce, les génotypes n'apparaissent pas se manifester " mieux " dans ce milieu, mais " différemment». Il est difficile de trancher, en ce qui concerne cet exemple, l'alternative " sélection en milieu favorable " ou " sélection en milieu défavorable "discutée par Hammond (8) et Falconer et LATYSZEFWSKI (4).

Si d'autre part on considère les estimations globales de l'héritabilité de la maturité sexuelle, portant sur la période entière d'éclosion, on voit que l'estimation $h_{d}^{2}$ (par la composante mère) prend une valeur moyenne de 0,25 , voisine des estimations courantes de la littérature, dont beaucoup ont été rassemblées par JULL (I3). Il est possible, si nos observations se trouvent vérifiées en d'autres circonstances, que l'influence de l'époque d'entrée en ponte rende compte en partie des différences entre les estimations d'héritabilité données par divers auteurs. Quant à $h_{s}^{2}$, il se trouve augmenté ici par le fait que la moitié des pères correspond à une période d'éclosion différente de l'autre, d'où une différence entre les deux séries de pères, causée par le milieu.

\section{c) Conséquences pratiques possibles}

$\mathrm{Si}$, encore une fois, ces résultats sont confirmés dans d'autres troupeaux, des conséquences pratiques intéressantes en découlent.

L'intérêt d'une maturité sexuelle convenable, maximum ou optimum, n'étant pas niable, l'utilisateur d'un troupeau commercial peut rechercher deux choses : ou bien obtenir de ses poulettes une bonne précocité sexuelle, en les faisant naître à une date déterminée; ou bien avoir des animaux qui entreront en ponte à un âge convenable, quelle que soit leur date d'éclosion - cas probablement le plus général, car il n'est pas toujours possible ou désirable de faire éclore sur une période trop limitée.

La conduite de la sélection dépendra du but recherché. Dans le premier cas, une sélection portant sur une périođe d'éclosion pédigree restreinte pourrait être plus efficiente que si cette période est longue, comme le mentionnent SKaLLER et SHELdON (20).

Si la période d'éclosion choisie a lieu tôt au printemps, les résultats présents indiquent que, la maturité sexuelle étant alors assez héritable, on peut pratiquer unes élection familiale, parfamilles de mère ou de père, ou même une sélection individuelle, si l'héritabilité atteint o,4-o,5 (L,ERNER, I6, page 2I2).

Quant aux poulettes nées plus tard, la sélection familiale serait seule possible ; on pourrait envisager une sélection par familles de demi-søeurs de même père, ou par progeny-test portant sur le choix des coqs. 
Mais une sélection ainsi conduite risque d'aboutir à des animaux qui n'auront une précocité convenable que s'ils sont nés à une certaine époque. Il apparait donc intéressant de rechercher s'il existe des génotypes de bonne maturité sexuelle pour un large éventail de dates d'éclosion, c'est-à-dire des animaux qui aient des aptitudes héréditaires à une maturité sexuelle précoce, et qui soient en même temps " résistants" aux conditions de nature à retarder l'entrée en ponte.

A première vue, c'est en faisant naître tard, pour provoquer l'entrée en ponte en saison "défavorable ", qu'on pourra sélectionner sur cette " résistance " vis-à-vis des conditions de milieu. Mais il est malaisé de distinguer entre les animaux dont la maturité serait tardive en n'importe quelle saison et ceux qui sont " retardés ". On peut alors penser à comparer les performances d'animaux parents à diverses époques de l'année. On pourrait concevoir, par exemple, le choix par progeny-test de coqs dont les descendantes, nées à des périodes d'éclosion successives, ont des précocités aussi voisines que possible.

\section{d) Recherches ultérieures}

Dans la transmission héréditaire des aptitudes à la ponte et son utilisation par la sélection, la sensibilité ou la résistance à des conditions variables de l'environnement sont donc un facteur à considérer. Son étude dans les conditions naturelles est d'une grande complexité, si l'on songe à toutes les variations simultanées, de durée du jour, de température et autres, qui accompagnent le changement des saisons. Aussi le conditionnement à volonté de facteurs extérieurs, tels que la lumière, peut-il permettre une connaissance expérimentale plus précise du mode de réponse de l'animal à ces facteurs, en fonction de sa constitution héréditaire. Cette étude en milieu partiellement conditionné peut, après transposition dans les conditions naturelles, conduire à de nouvelles applications pratiques dans la sélection pour la ponte.

\section{RESUME}

Sur 7 générations appartenant à deux troupeaux différents, élevés dans des conditions courantes en pratique, et sans emploi de lumière artificielle, 1'héritabilité de l'âge au premier œuf a été estimée séparément sur les poulettes écloses au début et à la fin de la saison pedigree du printemps. Les méthodes d'estimation incluaient l'analyse de la variance entre groupes de sœurs et de demi-sœurs, et, accessoirement, la régression filles-mères pour le même père.

Les résultats obtenus font ressortir chez les premières couvées une plus grande proportion de va riance génotypique par rapport à la variance totale, telle qu'elle est estimée d'après la " composante mère " de la 
variance, correspondant à l'action des gènes autosomaux. Ils suggèrent par contre la présence de gènes liés au sexe conditionnant la maturité sexuelle, et ne se manifestant pleinement que chez les derniers lots d'éclosion.

L'examen des distributions de fréquences des dates d'entrée en ponte s'accorde avec l'hypothèse antérieurement émise d'une influence défavorable, sur la maturité sexuelle, des jours rapidement décroissants.

La portée de ces résultats est discutée. D'un point de vue pratique, ils soulignent, dans des conditions analogues aux nôtres, l'intérêt d'une sélection par familles de pères, s'il s'agit de poulettes nées tard en saison. Pour les poulettes nées tôt, la sélection par familles de père ou de mère est possible, ou même la sélection individuelle. La comparaison des performances des filles d'un même père à des époques différentes de l'année pourrait aussi s'avérer intéressante, pour choisir les coqs dont la descendance dépend le moins des conditions saisonnières.

\section{Reçu pour publication le 8 mai 1956.}

\section{RÉFÉRENCES BIBLIOGRAPHIQUES}

(I) Abplanalp (H.). - The relative importance of environment in the evaluation of large families. Poul. Sci., 32, p. 885, I953.

(2) BYerLy (T. C.) et KNox (C. W.) - Date of hatch and day length affect age at first egg. Poul. Sci., 25, p. 587-592, I946.

(3) Cochez (L. P.). - Communications personnelles.

(4) FALCONER (D. S.) et LATYSZEWSKI (M.). - The environment in relation to selection for size in mice. $J$. Genetics, 51, p. 67-80, r952.

(5) Gonzalez-Chapel, (A.) et Rojas-Daporta (M.). - Influence of time of hatching on sexual maturity of pullets in Puerto-Rico. Jour. of Agric. Univ. of Puerto-Rico, 35, 3, p. IOo-II3, I95I.

(6) Greenwood (A. W.) et BiyTh (J. S. S.). - Sexual maturity in Brown Leghorns. Poul. Sci., 25, p. 597-605. I946.

(7) Haldane (J. B. S.). - The interaction of nature and nurture. Ann. Eugen. Cambr., 13, p. I97-205, I946.

(8) Hammond (J.). - Animal breeding in relation to nutrition and environmental conditions. Biol. Rev., 22, p. I95-213, I947.

(9) Hays (F. A.). - Inbreeding in the R. I. R. fowl with special reference to winter egg production. Amer. Naturalist, 58, p. 43-59, I924.

(io) Hazei, (L. N.) et I,Amoreux (W. F.). - Heritability, maternal effects and nicking in relation to sexual maturity and body weight in White Leghorns. Poul. Sci., 26, p. 508-5I4, I947.

(II) Hu'TT (F. B.). - Genetics of the fowl. McGraw Hill Book Co N. Y., Ire ed., 590 p., I949, p. 209-2II.

(I2) JEFFREY (F. P.) et PLATT (C. S.). - A 3-year study of out-of-season hatching. New Jersey Agr. Exp. Sta. Bul., 687, I94I.

(13) Jull (M. A.). - Poultry Breeding. Wiley and sons. N. Y., $3^{\text {e }}$ ed., 398, p. 296-300, 1952.

(I4) KING (S. C.) et HENDERSON (C. R.). - Variance components analysis in heritability studies. Poul. Sci., 33, p. I47-I54, I954. 
(I5) Korkman (N.). - Causes of variation in the size and weight of litters from sows. Acta Agric. Suec., 2, p. 253-310, I946-I947.

(I6) LERNER (I. M.). - Population genetics and animal improvement. Cambridge Univ. Press., 342 p., I950.

(I7) LERNER (I. M.) et CRUDEN (D.). - The heritability of egg weight : The advantages of mass selection and of early measurements. Poul. Sci., 30, p. 34-4I, I95I.

(I8) ONishi (N.). - On the inheritance of sexual maturity in single comb White Leghorns. Xe Congrès Mondial d'Aviculture, Sect. A, p. 30-33, 1954 .

(I9) OSBORNE (R.). - Sexual maturity in Brown Leghorns. The interactions of genotype and environment. Proc. Roy. Soc. Edinb. Sect. B, 54, p. $445-455$, I 952 .

(20) SkAliER (F.) et SHELDON (B. L.). - Interactions of genotype and environment on determining sexual maturity in the domestic fowl. Austr. J. of Agric. Res., 6, p. I7I-I 85, I954.

(2I) TAYLOR (L. W.) et LERNER (I. M.). - Breeding for egg production. Calif. Agr. Exp. Sta. Bul., 626, I938.

(22) UpP (C. W.) et Thompson (R. B.). - Influence of time of hatch on hatchability of the eggs, rate of growth of the chicks, and characteristics of the adult female. Oklahoma Agr. Exp. Sta. Bul., I67, I927.

(23) Vessereau (A.). - Méthodes statistiques en biologie et en agronomie. J. B. Baillière et Fils, 38I p,. I948.

(24) Warren (D. C.). - Crossbred poultry. Kansas Agr. Exp. Sta. Bul., 252, I930.

(25) WARREN (D. C.). - Inheritance of age at sexual maturity in the domestic fowl. Genet., I9, p. 600-6I7, I934.

(26) Whatrey (J. A. jr). - Influence of heredity and other factors on I80day weight in Poland-China swine. J. Agr. Res., 65, p. 249-264, 1942.

(27) Winsor (C. P.) et Clarke (G. L.). - A statistical study of variation in the catch of Plankton nets. J. marine Res., 3, I-34, I940. 